\title{
IMPREGNAÇÃO DO ÁCIDO 12-FOSFOTÚNGSTICO EM SÍLICA - PARTE I: DETERMINAÇÃO DE PARÂMETROS DE IMPREGNAÇÃO, CARACTERIZAÇÃO E AVALIAÇÃO DA ATIVIDADE CATALÍTICA
}

\author{
Karine Isabel Scroccaro*, Valcineide Oliveira de Andrade Tanobe, Lílian Cristina Côcco e Carlos Itsuo Yamamoto \\ Departamento de Engenharia Química, Universidade Federal do Paraná, Centro Politécnico, CP 19011, 81531-980 Curitiba - PR, \\ Brasil \\ Fernando Wypych \\ Departamento de Química, Universidade Federal do Paraná, Centro Politécnico, CP 19011, 81531-980 Curitiba - PR, Brasil
}

Recebido em 13/9/11; aceito em 12/3/12; publicado na web em 2/7/12

\begin{abstract}
IMPREGNATION OF 12-TUNGSTOPHOSPHORIC ACID ON SILICA - PART I: DETERMINATION OF IMPREGNATION PARAMETERS, CHARACTERIZATION AND EVALUATION OF CATALYTIC ACTIVITY. Catalyst based on Keggingtype heteropolyacids $\left(\mathrm{H}_{3} \mathrm{PW}_{12} \mathrm{O}_{40}\right.$ - HPA), supported on $\mathrm{SiO}_{2}\left(\mathrm{H}_{3} \mathrm{PW}\right)$, were prepared by the impregnation method under different thermal treatment conditions. The materials were characterized by different instrumental techniques and used as catalysts in the methyl esterification reactions of stearic acid. Using the catalyst with $15 \%$ of HPA, conversions higher than $60 \%$ were obtained after $2 \mathrm{~h}$ of reaction at $65^{\circ} \mathrm{C}$. Recovery studies using hot-filtration with ethanol at $75{ }^{\circ} \mathrm{C}$ showed satisfactory activity for two additional reaction cycles.
\end{abstract}

Keywords: esterification; tungstophosphoric acid; silica.

\section{INTRODUÇÃO}

Reações químicas catalíticas vêm sendo estudadas ao longo dos anos em distintas rotas de síntese, como forma de obtenção de produtos com altas taxas de conversão e minimização de custos. Atualmente, os aspectos da sustentabilidade têm impulsionado o desenvolvimento de novos materiais catalíticos suportados em matrizes sólidas, uma vez que existe a possibilidade de reuso destes materiais.

Entre estes catalisadores, os heteropoliácidos (HPAs) têm despertado grande interesse devido a suas propriedades, como baixa volatilidade, alta reatividade e seletividade, além da possibilidade de sua utilização em reações distintas, com destaque para o ácido 12-fosfotúngstico $\left(\mathrm{H}_{3} \mathrm{PW}_{12} \mathrm{O}_{40}\right.$ - HPA). Nestes HPAs, a fase ativa consiste na estrutura do ânion do tipo Keggin, o qual possui diâmetro em torno de 1,2 nm e é composto de um átomo central coordenado tetraedricamente a átomos de oxigênio, circundado de 12 unidades octaédricas, ligados por átomos de oxigênio vizinhos. O octaedro é organizado em quatro grupos $\mathrm{M}_{3} \mathrm{O}_{13}$, sendo que cada grupo é formado por três octaedros que compartilham seus vértices com um átomo de oxigênio comum ao tetraedro central $\mathrm{XO}_{4}$. O conjunto completo é composto de 40 grupos fechados de átomos de oxigênio. Os átomos de oxigênio estão ligados de duas maneiras: com ligação dupla $\mathrm{M}=\mathrm{O}$ e com ligação simples $\mathrm{M}-\mathrm{O}-\mathrm{M}$ unindo-se ao octaedro pelo grupo $\mathrm{M}_{3} \mathrm{O}_{13}$, no qual doze são ligados de uma forma quase linear $\mathrm{M}-\mathrm{O}-\mathrm{M}$ conectando-se a dois diferentes grupos $\mathrm{M}_{3} \mathrm{O}_{13}$, e quatro possuem ligações internas $\mathrm{X}-\mathrm{O}-\mathrm{M} .^{1-9}$

Atualmente uma série de estudos no desenvolvimento dessa classe de compostos tem sido voltada para a produção de ésteres, bem como em processos de heterogeneização de catalisadores que atuam em meio homogêneo utilizando suportes, tais como alumina, sílica, zircônia, entre outros. ${ }^{7,8}$

Nos distintos processos industriais a esterificação é uma reação importante, pois, a partir desta vários tipos de ésteres são produzidos para uso comercial, ${ }^{10}$ sendo um processo reversível, tendo como exemplo a reação de esterificação de Fischer (1895) com ácido sulfúrico em meio homogêneo. ${ }^{11}$ Entretanto, a recuperação do catalisador é

*e-mail: karineisabel@yahoo.com.br dificultada, por estar na mesma fase dos demais reagentes. Além disso, não é ambientalmente sustentável. ${ }^{1}$ Em consequência disso, muitos estudos têm sido realizados na busca por rotas alternativas não só para esterificação, mas para outros tipos de reações. Nesse sentido, vários estudos têm sido descritos quanto à imobilização de HPAs em vários suportes. Entre estes se podem citar esterificação do ácido mandélico, ${ }^{12}$ imobilização em sílica e zircônia e uso na esterificação do ácido acético com butanol, ${ }^{13}$ imobilização em alumina neutra e uso na esterificação do ácido fórmico, acético e propiônico, ${ }^{14}$ imobilização em zircônia hidratada e uso na obtenção de biodiesel. ${ }^{15}$ Outros trabalhos ainda relatam diversas formas de impregnações/ imobilizações de diversos HPAs em vários suportes e uso em reações variadas. ${ }^{16-21}$

Diante do exposto, este trabalho teve o objetivo de desenvolver e avaliar a aplicação de um catalisador à base de ácido 12-fosfotúngstico suportado em sílica em reações de esterificação metílica do ácido esteárico.

\section{PARTE EXPERIMENTAL}

\section{Impregnação do ácido fosfotúngstico em sílica}

O método utilizado no preparo do catalisador foi a impregnação por excesso de solução, ${ }^{16}$ no qual se utilizou uma sílica SI-1452 (Grace Davison) como suporte para impregnação do ácido 12-fosfotúngstico (Vetec) e o metanol (99,8\% - F. Maia). Todos os reagentes, exceto o metanol, foram submetidos a uma pré-secagem, sendo a sílica a 130 ${ }^{\circ} \mathrm{C}$ por $8 \mathrm{~h}$ e o HPA à $100{ }^{\circ} \mathrm{C}$ durante $6 \mathrm{~h}$.

As impregnações inicialmente foram realizadas em etapas e em triplicata (Tabelas 1 e 2), sendo que em cada uma delas foram estabelecidas as condições de secagem (modo A, B e C), temperaturas de calcinação $\left(400,450,500,530,550,570\right.$ e $\left.590{ }^{\circ} \mathrm{C}\right)$ e tempo de calcinação $(3 \mathrm{~h})$. Foram mantidas fixas as seguintes condições: proporção de água/metanol (1:1), tempo de impregnação (24 h), velocidade da agitação magnética $(150 \mathrm{rpm})$, massa do suporte $(5 \mathrm{~g})$ e massa do HPA ( $2 \mathrm{~g}$ ).

Os parâmetros utilizados como controle de qualidade da atividade catalítica do catalisador foram o teor de éster e a lixiviação do ânion Keggin no meio reacional. 
Tabela 1. Parâmetros térmicos versus teor de éster

\begin{tabular}{cccc}
\hline \multirow{2}{*}{$\begin{array}{c}\text { Temperatura de } \\
\text { calcinação }\left({ }^{\circ} \mathrm{C}\right)\end{array}$} & \multicolumn{3}{c}{ Teor de éster $(\%)$} \\
\cline { 2 - 4 } & Modo A & Modo B & Modo C \\
\hline 500 & 33 & 46 & 38 \\
450 & 95 & 86 & 72 \\
400 & 90 & 91 & 89 \\
350 & 89 & 89 & 76 \\
\hline
\end{tabular}

Obs: Modo de secagem A $\left(2 \mathrm{~h} \mathrm{a} 60^{\circ} \mathrm{C} ; 2 \mathrm{~h} \mathrm{a} 80^{\circ} \mathrm{C} ; 2 \mathrm{~h}\right.$ a $100{ }^{\circ} \mathrm{C} ; 2 \mathrm{~h}$ a $\left.120^{\circ} \mathrm{C}\right)$, Modo de secagem $\mathrm{B}\left(2 \mathrm{~h} \mathrm{a} 60^{\circ} \mathrm{C} ; 3 \mathrm{~h}\right.$ a $80^{\circ} \mathrm{C} ; 3 \mathrm{~h}$ a $\left.120^{\circ} \mathrm{C}\right)$ e Modo de secagem $\mathrm{C}\left(1 \mathrm{~h} \mathrm{a} 60{ }^{\circ} \mathrm{C} ; 3 \mathrm{~h}\right.$ a $80{ }^{\circ} \mathrm{C} ; 3 \mathrm{~h}$ a $100{ }^{\circ} \mathrm{C} ; 1 \mathrm{~h}$ a $\left.120^{\circ} \mathrm{C}\right)$.

Tabela 2. Teor de éster versus lixiviação

\begin{tabular}{ccc}
\hline $\mathrm{T}\left({ }^{\circ} \mathrm{C}\right) / 3 \mathrm{~h}$ & ${ }^{*}$ Éster $(\%)$ & ${ }^{* *}$ Lixiviação $(\mathrm{ppm})$ \\
\hline 590 & 29 & 2 \\
570 & 27 & 3 \\
550 & 78 & 7 \\
530 & 62 & 4 \\
500 & 100 & 11 \\
\hline
\end{tabular}

Nota: $*$ O desvio das medidas é $10 \%: * *$ desvio menor que $1 \mathrm{ppm}$

\section{Caracterização dos materiais}

A sílica, o ácido fosfotúngstico e o catalisador obtido na temperatura de calcinação de $530{ }^{\circ} \mathrm{C}\left(2 \mathrm{~g}\right.$ de $\mathrm{HPA}$ em $5 \mathrm{~g}$ de $\left.\mathrm{SiO}_{2}\right)$ foram previamente caracterizados em todas as etapas por distintas técnicas instrumentais. Os materiais antes de serem analisados, tanto por difração de raios X (DRX) como por fluorescência de raios X (FRX), foram previamente pulverizados em um moinho para se obter um diâmetro médio de partícula inferior a $0,044 \mathrm{~mm}$, conforme metodologia de análise utilizada.

A técnica de FRX foi utilizada para determinação dos elementos constituintes das amostras (expressas na forma óxidos - $\mathrm{WO}_{3} \mathrm{e}$ $\mathrm{P}_{2} \mathrm{O}_{5}$ ), antes e após o processo de impregnação a úmido. Nas medidas utilizou-se um equipamento Philips - PW 2400 Sample Changer com fonte de Rh. Após a análise por FRX, $2 \mathrm{~g}$ de amostra foram colocadas em estufa a $100{ }^{\circ} \mathrm{C}$ por $2 \mathrm{~h}$, para determinação da perda ao fogo (orgânicos).

A técnica de DRX foi utilizada para avaliar as distintas fases (cristalina e amorfa) formadas antes e após a impregnação, bem como acompanhar as características estruturais do catalisador após sua recuperação do meio reacional.

As propriedades texturais dos materiais (volume de poros e microporos, área dos poros e área superficial) foram determinadas por isotermas de adsorção/dessorção em $\mathrm{N}_{2}$ (BET), método BJH, sendo que as amostras foram previamente secas em estufa a vácuo a 110 ${ }^{\circ} \mathrm{C}$ por $3 \mathrm{~h} .{ }^{11} \mathrm{O}$ equipamento utilizado foi um porosímetro, marca Quantachrome, modelo Nova 1200.

\section{Atividade catalítica}

Os reagentes empregados na esterificação foram o ácido esteárico $\left(\mathrm{C}_{18} \mathrm{H}_{36} \mathrm{O}_{2}\right)$ (Merck - 97\%), metanol $\left(\mathrm{CH}_{3} \mathrm{OH}\right.$, Vetec - 99,8\%) e os distintos catalisadores obtidos conforme descritos anteriormente.

As reações de esterificação metílica do ácido esteárico foram realizadas em um evaporador rotativo (em triplicata: $2 \mathrm{~h} \mathrm{a} 65^{\circ} \mathrm{C}$ ), com distintas razões molares metanol/ácido esteárico (10:1,30:1 e 50:1) e concentrações do catalisador de 5, 15, 25 e 35\% (m/m, em relação à massa do ácido esteárico). Para comparar os teores obtidos de éster foram realizadas reações na ausência de catalisador, denominadas de brancos de reação ou conversões térmicas.

A quantificação do éster $\left(\mathrm{C}_{19} \mathrm{H}_{38} \mathrm{O}_{2}\right)$ foi realizada por cromatografia a gás $(\mathrm{CG})$ com um detector FID, coluna CP-SIL $5 \mathrm{CB}$, por um procedimento de calibração interna com metil-heptadecanoato em $\mathrm{CHCl}_{3}$. O teor de éster foi definido como a percentagem do estearato de metila obtido a partir da concentração inicial deste no meio reacional.

\section{Teste de lixiviação e recuperação do catalisador}

Após as reações de esterificação foram recolhidas alíquotas do meio alcoólico e determinadas as concentrações do HPA lixiviado por meio da espectrofotometria na região do UV/VIS, com um equipamento Varian Inc., modelo Cary 50 scan, com varredura de 800 a $200 \mathrm{~nm}$ e resolução de $1 \mathrm{~nm}$. O monitoramento da presença do HPA por UV-VIS baseia-se nas bandas de absorção em $220 \mathrm{~nm}$ (atribuída à transferência de carga ligante-metal) e a 265 nm (atribuída à transferência de carga ligante-metal). ${ }^{22}$

A recuperação dos catalisadores do meio reacional foi realizada por meio de filtração a quente (hot-filtration) com $50 \mathrm{~mL}$ de etanol a $75^{\circ} \mathrm{C}$, para remoção completa do éster, seguida de secagem em estufa a $100{ }^{\circ} \mathrm{C}$ por $4 \mathrm{~h}$. Após recuperação, os catalisadores foram caracterizados e reutilizados nas reações de esterificação para avaliar quantos ciclos de reuso estes suportariam sem o comprometimento da sua função ou atividade catalítica.

\section{RESULTADOS E DISCUSSÃO}

\section{Preparo dos catalisadores}

Entre os distintos métodos utilizados no preparo de catalisadores, o método da impregnação por excesso de solução foi utilizado por ser de fácil realização, embora a complexidade de parâmetros envolvidos seja grande. Deste modo, as etapas que envolvem a pré-secagem, calcinação, tempo de contato entre as fases e recuperação do catalisador foram estabelecidas com base na literatura. ${ }^{1-8}$ Quanto ao tempo de contato entre as fases, a opção por 24 h sob agitação magnética foi estabelecida como tempo suficiente para que ocorresse o equilíbrio que envolve os processos de impregnação. ${ }^{7}$

Entre os parâmetros térmicos estabelecidos inicialmente, o modo de secagem, a temperatura e o tempo de calcinação foram considerados relevantes no preparo do catalisador. Os três modos de secagem utilizados (A, B e C), bem como as temperaturas entre 350 e $450^{\circ} \mathrm{C}$ e tempo de $3 \mathrm{~h}$ de calcinação resultaram em teores de conversão de éster entre 72 a 96\%, conforme apresentado nas Tabelas 1 e 2. Entretanto, a lixiviação do HPA no catalisador preparado a $450{ }^{\circ} \mathrm{C}$ foi superior a 50 ppm (dados não apresentados), sendo assim, avaliou-se conjuntamente as taxas de conversão (Figura 1) e o teor de HPA lixiviado no meio reacional (Tabela 3 ). Com base nestes resultados, todos os outros catalisadores estudados foram preparados na temperatura de calcinação de $530{ }^{\circ} \mathrm{C}$, pois nessas condições foram obtidas as taxas de conversão de $62 \%$ e as taxas de lixiviação do HPA (4 ppm), sendo considerado o conjunto otimizado, conforme discussão posterior da atividade catalítica.

\section{Caracterização dos catalisadores}

A Figura 2 mostra os difratogramas da $\mathrm{SiO}_{2}, \mathrm{H}_{3} \mathrm{PW}_{12} \mathrm{O}_{40}$ e do catalisador $\left(\mathrm{SiO}_{2} / \mathrm{H}_{3} \mathrm{PW}\right)$. Observa-se que a sílica apresenta caráter amorfo, caracterizado por um halo amorfo na região entre 15 a $30^{\circ}$ (em 20).22 $\mathrm{O}$ difratograma do HPA evidencia caráter altamente cristalino, com distintos planos de difração. Este padrão de difração 


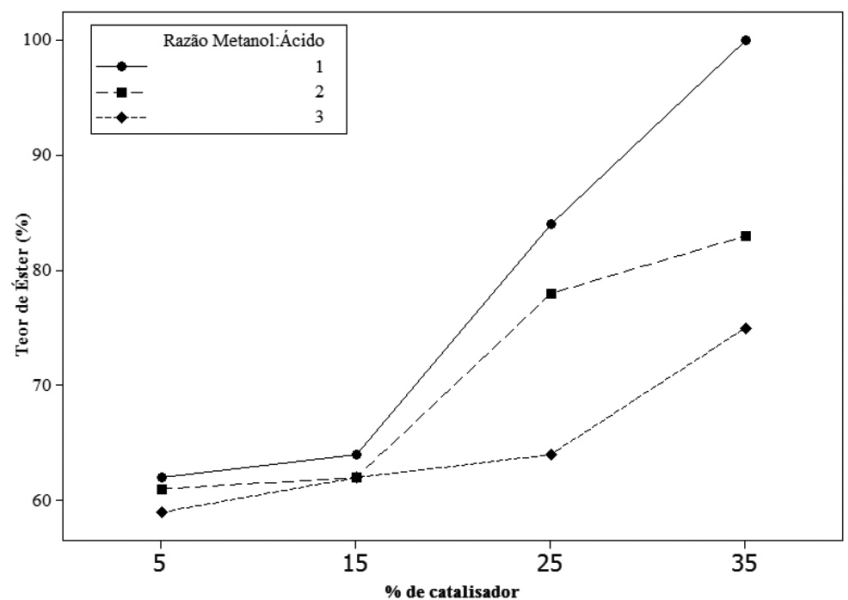

Figura 1. Teor de éster versus razão molar e \% de catalisador

Tabela 3. Lixiviação versus razão molar e \% de catalisador

\begin{tabular}{ccccc}
\hline & \multicolumn{4}{c}{$\%$ Catalisador $\left(530^{\circ} \mathrm{C}\right)$} \\
\cline { 2 - 5 } & 5 & \multicolumn{4}{c}{15} & 25 & 35 \\
\cline { 2 - 5 } & \multicolumn{5}{c}{${ }^{* *}$ Lixiviações $(\mathrm{ppm})$} \\
\hline $50: 1$ & 3 & 5 & 2 & 4 \\
$30: 1$ & 3 & 2 & 2 & 3 \\
$10: 1$ & 2 & $<1$ & $<1$ & 1 \\
\hline
\end{tabular}

Nota: **desvio menor que 1 ppm

é complexo devido às várias estruturas existentes no ânion Keggin, bem como às interações devido às formas de protonação deste ácido. O HPA hexa-hidratado apresenta estrutura cúbica, com planos de reflexão $\left(100,110,200,040\right.$ e 400). Plano de difração em $2=10^{\circ}$ (plano 100) é referente ao composto $\mathrm{H}_{3} \mathrm{PW}_{12} \mathrm{O}_{40}$ e em $2=26$ e $35^{\circ}$ tem-se o composto $\mathrm{H}_{3} \mathrm{PW}_{12} \mathrm{O}_{40}$, conforme também constatado em outros autores. ${ }^{22} \mathrm{O}$ difratograma do catalisador evidencia que a amostra apresenta características amorfas, o que atesta a boa dispersão do HPA na sílica, considerando-se que se houvesse segregação da alguma fase do HPA ou formação do $\mathrm{WO}_{3}$ seriam observados picos de difração característicos.

As propriedades texturais e a composição química são mostradas na Tabela 4. Pode-se verificar que o ácido possui uma área superficial muito pequena $\left(4,2 \mathrm{~m}^{2} \mathrm{~g}^{-1}\right)$, quando comparada à sílica $\left(333,0 \mathrm{~m}^{2} \mathrm{~g}^{-1}\right)$ e ao catalisador obtido $\left(279,0 \mathrm{~m}^{2} \mathrm{~g}^{-1}\right)$. Os outros parâmetros, tais como área de poros, volume de poros e volume de microporos, diminuem ligeiramente no catalisador obtido, quando comparados à sílica. Isto se deve à impregnação pelo ácido, o qual está distribuído na estrutura

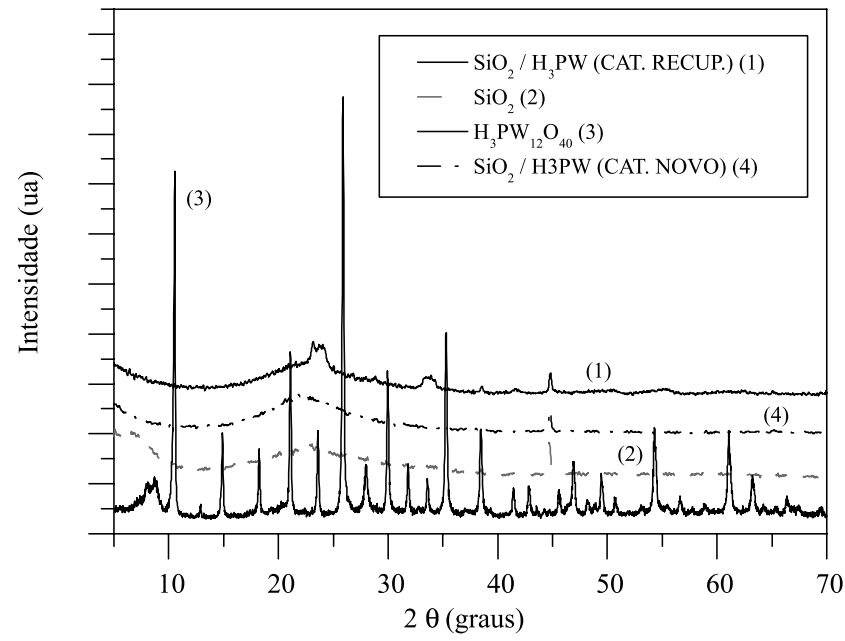

Figura 2. Difratogramas de raios $\mathrm{X}$ da $\mathrm{SiO}_{2}, \mathrm{H}_{3} \mathrm{PW}_{12} \mathrm{O}_{40}$ e do catalisador $\left(530{ }^{\circ} \mathrm{C}-5 \mathrm{~g}\right.$ de $\mathrm{SiO}_{2} / 2 \mathrm{~g}$ de $\left.\mathrm{H}_{3} \mathrm{PW}_{12} \mathrm{O}_{40}\right)$

do material, bem como devido à formação do coque durante a calcinação, que pode obstruir os poros, conforme descrito na literatura. ${ }^{22}$

A análise por FRX (Tabela 4) mostrou que no catalisador obtido o teor de $\mathrm{SiO}_{2}$ diminui com relação ao suporte, de 93 para $71 \%$, indicando que houve a impregnação pelo ácido, devido à presença dos elementos constituintes do HPA. Neste caso, o catalisador apresentou um teor de $21 \%$ de $\mathrm{WO}_{3}$ e $1 \%$ de $\mathrm{P}_{2} \mathrm{O}_{5} \cdot{ }^{18}$ Os cálculos referentes ao teor de impregnação do HPA na sílica foram realizados com base em dados de área superficial da sílica, conforme descrito na Parte Experimental; além das informações do diâmetro médio e da massa molecular do ácido, foi possível calcular a razão ácido:sílica na proporção utilizada de $2 \mathrm{~g}$ de $\mathrm{H}_{3} \mathrm{PW}_{12} \mathrm{O}_{40}$ para $5 \mathrm{~g}$ de $\mathrm{SiO}_{2}$, como correspondente a $51 \%$ de $\mathrm{H}_{3} \mathrm{PW}$ e $49 \%$ de $\mathrm{SiO}_{2}{ }^{23}$

\section{Reações de esterificação metílica do ácido esteárico}

A avaliação da atividade catalítica foi quantificada pelo teor do estearato de metila obtido com as distintas razões de metanol/ácido esteárico. O teor de éster obtido na ausência de catalisador foi somente $3 \%$ na maior razão molar álcool/ácido esteárico, conforme já esperado e também constatado na literatura. ${ }^{15} \mathrm{~A}$ Figura 1 evidencia as seguintes situações: o teor de conversão de aproximadamente $60 \%$, praticamente não se altera com o percentual de catalisador em 5 e $15 \%$ em todas as razões molares avaliadas; o aumento da concentração de catalisador entre 25 e $35 \%$ promoveu maior teor de éster nas maiores razões molares metanol/ácido, diminuindo na menor razão (5 a 10\%). Na Tabela 3, o teor de lixiviação é sempre menor em menor razão molar para todos os casos. Sendo assim, a melhor combinação

Tabela 4. Composição e propriedades da sílica, HPA e catalisador

\begin{tabular}{|c|c|c|c|c|c|}
\hline \multicolumn{6}{|c|}{ Propriedades texturais } \\
\hline Amostra & área superficial $\left(\mathrm{m}^{2} \mathrm{~g}^{-1}\right)$ & área microporos $\left(\mathrm{m}^{2} \mathrm{~g}^{-1}\right)$ & vol. poros $\left(\mathrm{cc} \mathrm{g}^{-1}\right)$ & vol. microporos $\left(\mathrm{cc} \mathrm{g}^{-1}\right)$ & diâmetro médio poros $(\AA)$ \\
\hline $\mathrm{SiO}_{2}$ & 333,0 & 458,0 & 1,0 & 0,1 & 123,0 \\
\hline HPA & 4,2 & 4,9 & $5,6.10^{-3}$ & $1,1.10^{-3}$ & 30,1 \\
\hline Catalisador & 280,0 & 386,0 & 0,9 & 0,1 & 124,0 \\
\hline \multicolumn{6}{|c|}{${ }^{* * * *}$ Composição química } \\
\hline Amostra & $\mathrm{SiO}_{2}(\%)$ & $\mathrm{WO}_{3}(\%)$ & $\mathrm{P}_{2} \mathrm{O}_{5}(\%)$ & $\mathrm{CaO}(\%)$ & Perda ao fogo (\%) \\
\hline $\mathrm{SiO}_{2}$ & 93 & - & - & $<1$ & 6 \\
\hline Catalisador & 71 & 21 & 1 & $<1$ & 6 \\
\hline
\end{tabular}

Nota: $* * *$ desvio da medida menor que $10 \%$ 
Tabela 5. Composição e propriedades do catalisador recuperado

\begin{tabular}{cccccc}
\hline \multicolumn{5}{c}{ Propriedades texturais } \\
\hline Amostra & área superficial $\left(\mathrm{m}^{2} \mathrm{~g}^{-1}\right)$ & área microporos $\left(\mathrm{m}^{2} \mathrm{~g}^{-1}\right)$ & vol. poros $\left(\mathrm{cc} \mathrm{g} \mathrm{g}^{-1}\right)$ & vol. microporos $\left(\mathrm{cc} \mathrm{g} \mathrm{g}^{-1}\right)$ & diâmetro médio poros $(\AA)$ \\
Catalisador recuperado & 282,0 & 384,0 & 0,9 & 0,1 & 124,0 \\
\hline Amostra & $\mathrm{SiO}_{2}$ & ${ }^{* * *}$ Composição Química (\%) & $\mathrm{CaO}$ & Perda ao fogo \\
\hline Catalisador recuperado & 75 & $\mathrm{WO}_{3}$ & $\mathrm{P}_{2} \mathrm{O}_{5}$ & $<1$ & 4 \\
\hline
\end{tabular}

Nota: $* * *$ desvio da medida menor que $10 \%$

dos parâmetros estipulados (\% de éster e \% de HPA lixiviado) foi obtida para a reação com $15 \%$ de catalisador e razão molar metanol/ ácido esteárico de 10:1, sendo obtida uma taxa de lixiviação de 1 ppm do HPA. ${ }^{24}$ Essa etapa foi necessária para avaliar a desativação do catalisador e para estudo posterior do reuso.

\section{Estabilidade e recuperação dos catalisadores}

A estabilidade destes catalisadores geralmente é avaliada em relação à lixiviação da fase ativa no meio reacional. Os resultados obtidos são mostrados na Figura 3, onde somente em 35\% de catalisador ocorreu alto teor de lixiviação (Tabela 3). Provavelmente o catalisador não está completamente estabilizado, sendo ligeiramente lixiviado no meio reacional, pois o HPA é pouco solúvel em metanol. ${ }^{24}$ Na concentração de $15 \%$ de catalisador e razão molar metanol/ácido esteárico de 10:1 praticamente não se verifica a lixiviação da fase ativa, pois não há evidências das bandas nas regiões de 220 e 265 nm, conforme se observa na Figura 3.

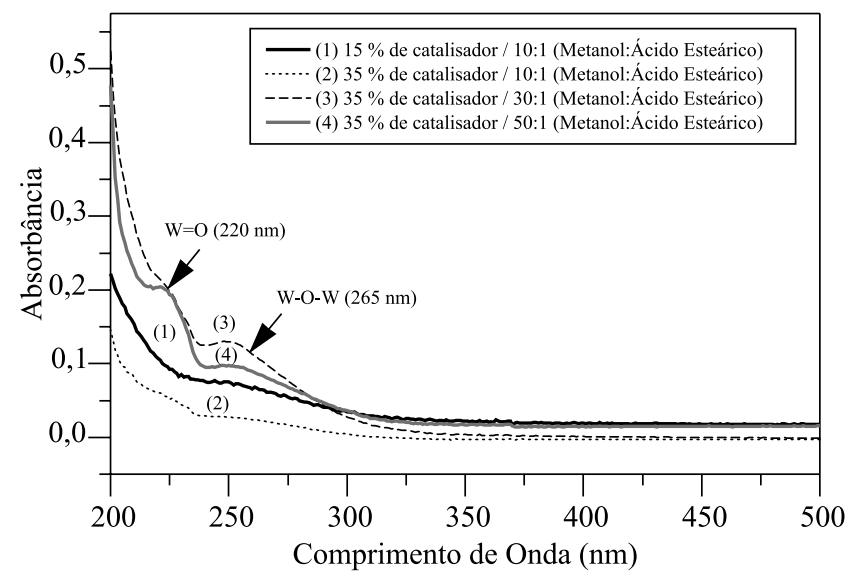

Figura 3. Espectro de UV-VIS do lixiviado do meio alcoólico (catalisador $530{ }^{\circ} \mathrm{C}-2 \mathrm{~g}$ de $\mathrm{SiO}_{2} / 5 \mathrm{~g}$ de $\mathrm{H}_{3} \mathrm{PW}_{12} \mathrm{O}_{40}$ )

Após a recuperação em etanol verificou-se que todos os parâmetros químicos e texturais permaneceram praticamente inalterados, conforme observado nas Tabelas 4 e 5 . Esta constatação é um fator positivo, indicando estabilidade mecânica e química do catalisador. ${ }^{18}$

A capacidade de reuso foi avaliada em três ciclos reacionais e, de acordo com a Figura 4, há uma pequena desativação do catalisador em cerca de $10 \%$ quando comparada à conversão do éster em $62 \%$ no primeiro uso. Além disso, o teor de HPA lixiviado mantém-se praticamente constante $(<1 \mathrm{ppm})$. Estes resultados são superiores aos encontrados em Santos et al., ${ }^{22}$ para um catalisador de mesma categoria, no qual se obteve alta desativação. $O$ fenômeno de desativação geralmente é atribuído à obstrução dos poros do catalisador, devido aos produtos de reação e à formação do coque durante o processo de calcinação do catalisador. ${ }^{24}$

Além da avaliação das propriedades texturais, composição

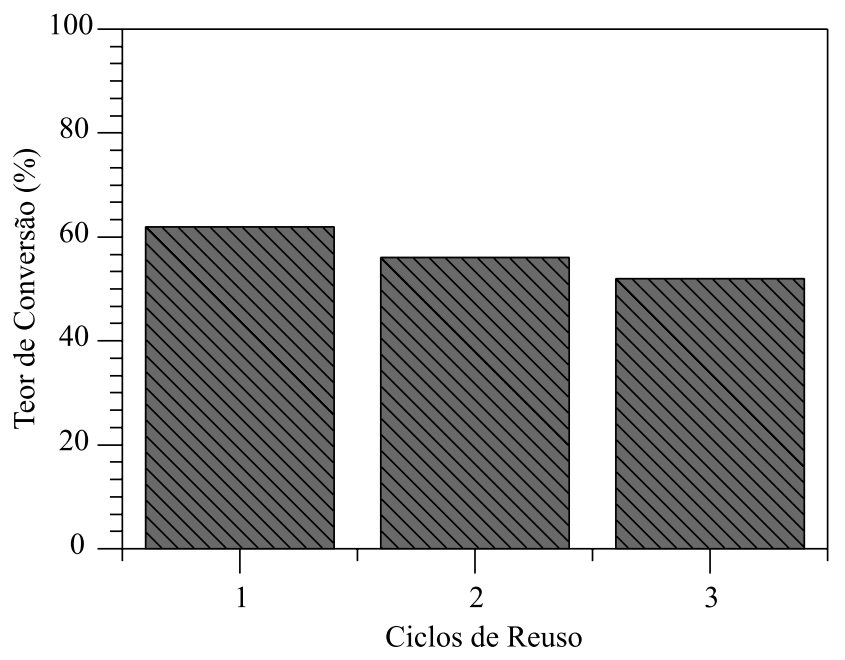

Figura 4. Ciclos de reuso versus teor de éster

química e teor de lixiviação, o catalisador recuperado foi também avaliado por DRX. A Figura 2 mostra o difratograma do catalisador após limpeza com etanol. O padrão de difração evidencia a prevalência de uma fase amorfa, com pequenos picos de difração que não podem ser atribuídos ao $\mathrm{H}_{3} \mathrm{PW}_{12} \mathrm{O}_{40}$ e sim ao seu produto de decomposição térmica $\left(\mathrm{WO}_{3}\right){ }^{25}$

Todos os parâmetros antes e após recuperação indicam boa estabilidade e capacidade de reuso, pois não há comprometimentos estrutural, mecânico e químico do catalisador, indicados pela manutenção da atividade catalítica em média de 54\%, conforme também verificado em Dias et al... ${ }^{25}$

\section{CONCLUSÕES}

O desenvolvimento do catalisador suportado nas condições estipuladas neste estudo possibilitou a obtenção de teores de estearato de metila acima de $60 \%$, sendo satisfatório em termos de recuperação e de manutenção da atividade catalítica. O melhor conjunto de resultados foi conseguido com 15\% de catalisador e uma razão molar de 10:1 de metanol/ácido esteárico. Foi possível também a recuperação desse catalisador em solvente de baixo custo e sua posterior reutilização em mais de 2 ciclos sem o comprometimento da atividade catalítica, uma vez que as características físico-químicas e texturais permaneceram nos mesmos níveis antes e após a recuperação, indicando assim a estabilidade do catalisador no meio reacional.

\section{AGRADECIMENTOS}

À Coordenação de Aperfeiçoamento de Pessoal de Nível Superior - CAPES, pela bolsa. Ao Laboratório de Análise de Minerais e Rochas - LAMIR e ao Instituto de Tecnologia para o Desenvolvimento LACTEC pelo suporte técnico nas análises de caracterização dos materiais. À G. Davison, pela doação da sílica. 


\section{REFERÊNCIAS}

1. Ivanov, A. V.; Vasina, T. V.; Nissenbaum, V. D.; Kustov, L. M.; Timofeeva, M. N.; Houzvicka, J. I.; Appl. Catal., A 2004, 259, 65.

2. Juan, J. C.; Zhang, J.; Yarmo, M. A.; J. Mol. Catal. A: Chem. 2007, 267, 265.

3. Nandhini, K. U.; Arabindoo, B.; Palanichamy, M.; Murugesan,V.; J. Mol. Catal. A: Chem. 2006, 243, 183

4. Patel, S.; Purohit, N.; Patel, A.; J. Mol. Catal. A: Chem. 2003, 192, 195.

5. Mallik, S.; Dash, S. S.; Parida, K. M.; Mohapatra, B. K.; J. Colloid Interface Sci. 2006, 300, 237.

6. Ferrell III, J. R.; Kuo, M. C.; Turner, J. A.; Herring, A. M.; Electrochim. Acta 2008, 53, 4927.

7. Caetano, C. S.; Fonseca, I. M.; Ramos, A. M.; Vital, J.; Castanheiro, J. E.; Catal. Commun. 2008, 9, 1996.

8. Kozhevnikov, I. V.; Catalysts for Fine Chemical Synthesis-Catalysis by Polyoxometalates, Wiley: England, 2002, vol. 2.

9. Fedotov, M. A.; Maksimovskaya, R. I.; J. Struct. Chem. 2006, 47, 952.

10. Phalak, C. A.: Tese de Doutorado, University of Pune, Índia, 2004.

11. Chimienti, M.; Pizzio, L. R.; Cáceres, C. V.; Blanco, M. N.; Appl. Catal., A 2001, 208, 7.

12. Rafiee, E.; Joshaghani, M.; Tork, F.; Fakhri, A. Eavani, S.; J. Mol. Catal. A: Chem. 2008, 283, 1.
13. Sepúlveda, J. H.; Yori, J. C.; Vera, C. R.; Appl. Catal., A 2005, 288, 18.

14. Sharma, P.; Vyas, S.; Patel, A.; J. Mol. Catal. A: Chem. 2004, 214, 281.

15. Sunita, G.; Devassy, B. M.; Vinu, A.; Sawant, D. P.; Balasubramanian, V. V.; Halligudi, S. B.; Catal. Commun. 2008, 9, 696.

16. Regalbuto, J.; Catalyst Preparation, CRC Press: New York, 2007.

17. Pizzio, L. R.; Cáceres, C. V.; Blanco, M. N.; Appl. Catal., A 1998, 167, 283.

18. Kozhevnikov, I. V.; J. Mol. Catal. A: Chem. 2007, 262, 86.

19. Lana, E. J. L.; Rocha, K. A. da S.; Kozhevnikov, I. V.; Gusevskaya, E. V.; J. Mol. Catal. A: Chem.2006, 259, 99.

20. Dupont, P.; Lefebvre, F.; J. Mol. Catal. 1996, 114, 299.

21. Lebedev, N. N.; Chemistry and Technology of Basic Organic and Petrochemical Synthesis, Mir Publishers: Moscou, 1984.

22. Santos, G. L. G.; Silva, C. S.; Cardoso, L. A. M.; Quim. Nova 2010, 33, 57.

23. Dias, J. A.; Rangel, M. do C.; Dias, S. C. L.; Caliman, E.; Garcia, F. A. C.; Appl. Catal., A 2007, 328, 189.

24. Kozhevnikov, I. V.; Appl. Catal., A 2003, 256, 3.

25. Dias, A. J.; Caliman, E.; Dias, S. C. L.; Paulo, M. A.; Thyrso, C. P. de S.; Catal. Today 2003, 85, 39. 